\title{
Two-Step Registration of Subacute to Hyperacute Stroke MRIs
}

\author{
P. Anbeek ${ }^{1}$, K.L. Vincken ${ }^{1}$, M.J.P. van Osch ${ }^{1}$, J.P.W. Pluim ${ }^{1}$, J. van der Grond ${ }^{1}$, \\ M.A. Viergever ${ }^{1}$ \\ ${ }^{1}$ Department of Radiology, Image Sciences Institute, University Medical Center, \\ Heidelberglaan 100, rm E01.334, Utrecht, The Netherlands \\ \{nelly, koen, thijs, josien, jeroen, max\}@isi.uu.nl
}

\section{Introduction}

In the use of MRI in acute stroke patients, determination of the perfusion-diffusion (PWI-DWI) mismatch is highly important, since it indicates possible enlargement of the ischemic lesion or deterioration of the patient. When quantitative measures are used such as the ADC of CBF to predict ischemic damage, accurate registration of the ischemic lesion on follow-up MRI to the results obtained in the first MRI is essential. Problems in the registration of stroke images include differences in patient orientation and/or low cooperativeness of the patient in the hyperacute stage of stroke. The aim of the present study is to develop a robust method of stroke image registration.

\section{Methods}

Registration was performed by Mirit (1), which is based on mutual information. Mirit provides rigid body registration, including rotation, translation and scaling. The image that is modified is the floating image, whereas the image to which the registration is performed is the reference image. We used the MRI data sets of 6 stroke patients, in whom the hyperacute dataset was obtained within 3 hours after the onset of stroke ( 0 ) and at 1 week (t1). Two ways of image registration were compared:

1. One-step: Direct registration of a floating image at t1 (FLAIR, T2-w or PD) to the reference image of different type at t0 (DWI or ADC-map).

2. Two-step: First performing a registration of an image at t1 (FLAIR, T2-w or PD) to an image of the same type at $\mathrm{t} 0$. The parameters for translation, rotation and scaling resulting from this registration are used as starting position for the second registration: Registration of the floating image at $t 1$ to a reference image of different type at $\mathrm{t} 0$ (DWI or ADC-map).

An example (FLAIR to DWI) of both image registration methods is shown in figure 1. The left side of the picture shows the registration by the one-step method. The right side shows registration by the two-step method. The white line in the picture denotes the contours of the cortex in the DWI scan, which is the reference image. These pictures indicate that for these images the registration and in particular scaling is performed better by the two-step method.

W. Niessen and M. Viergever (Eds.): MICCAI 2001, LNCS 2208, pp. 1279-1280, 2001.

(C) Springer-Verlag Berlin Heidelberg 2001 


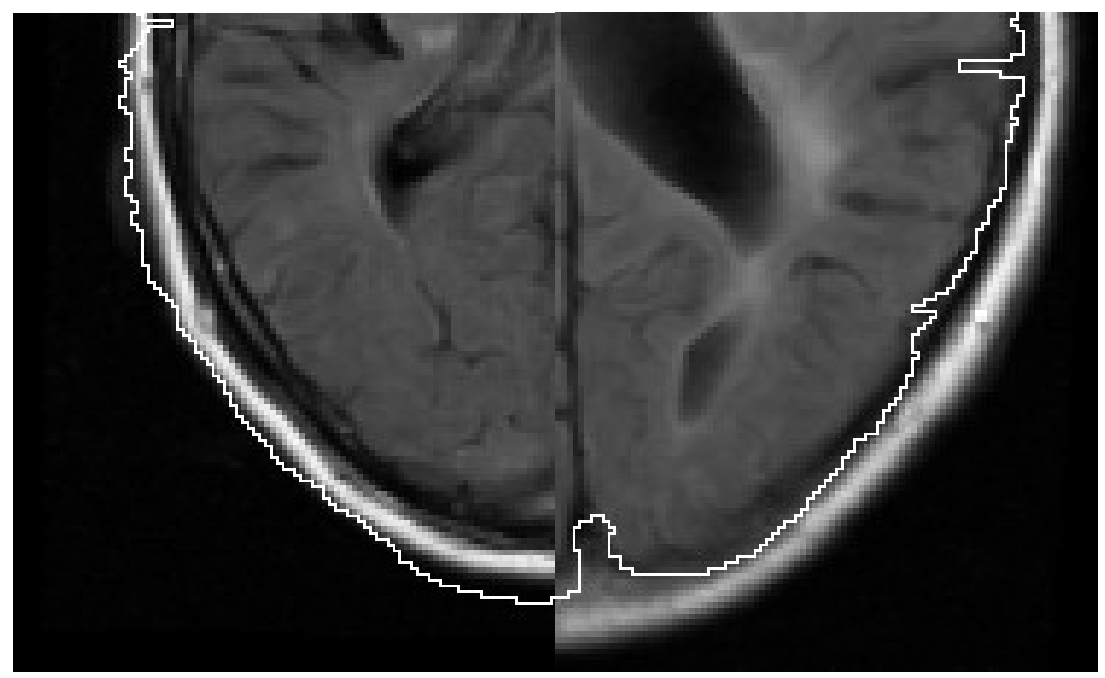

Figure 1: one-step versus two-step registration FLAIR to DWI

\section{Results}

In total we compared 72 registrations, 36 registrations by the one-step method with 36 corresponding registrations by the two-step method. Overall, the two-step method yielded in $72 \%$ a better registration ( $\mathrm{p}<0.001$, Fisher's exact test) when the DWI scan was used as reference image and in 39\% ( $\mathrm{p}<0.01)$ when the ADC-map was used as reference image, compared with the one-step method.

\section{Discussion}

The most important finding of the present study is that the two-step registration method is superior to direct registration of follow-up MRI stroke scans to hyperacute stroke scans. Our study has one major limitation. For practical reasons we applied registration by rotation, translation and scaling. It is well known that the current echo planar imaging (EPI) scans may distort the geometry of the brain. Elastic registration, in which is corrected for these image distortions, is the preferred way of registration. However, such a way of image registration is significantly more time consuming.

\section{References}

1. F. Maes et al., IEEE TMI 1997, 16, 187-198. 